Apidologie, 1983, 14 (1), 9-27.

\title{
PRÜFUNG DER WIRKUNG VON FOLBEX-VA (ISOPROPYL-4, 4'-DIBROMBENZILAT) AUF VARROAMILBEN UND DER VERTRÄGLICHKEIT FÜR BIENEN
}

\author{
Wolfgang RITTER, Friedrich PERSCHIL \\ Tierhygienisches Institut Freiburg, D-7800 Freiburg
}

\begin{abstract}
ZUSAMMENFASSUNG
Isopropyl-4, 4-dibrombenzilat ist als Wirkstoff im FOLBEX-VA-Räucherstreifen enthalten, der von der Ciba Geigy AG in Zusammenarbeit mit dem Tierhygienischen Institut Freiburg entwickelt wurde. In den durchgeführten Labor- und Freilandversuchen erwies sich FOLBEX-VA als gut bienen- und brutverträglich. Die Wirkung auf Varroamilben wurde anhand von 84 abgetöteten Völkern nachgewiesen. Mit einer 2 maligen Anwendung von je einem Räucherstreifen pro Volk im Abstand von 4 Tagen kann selbst in Völkern mit Brut ein Befall von mehr als 5 Milben sicher nachgewiesen werden. Mit einer 4 maligen Anwendung können im Herbst in Völkern ohne Brut über $97 \%$ der Milben abgetötet werden. FOLBEX-VA erfüllt somit die Voraussetzungen, die an ein Medikament zur Bekämpfung der parasitären Bienenmilben, Varroa jacobsoni, gestellt werden.
\end{abstract}

\section{EINLEITUNG}

Die Varroatose ist zur Zeit eines der größten Probleme der Weltbienenzucht. Der Erreger, die Milbe Varroa jacobsoni Oudemanns, parasitiert auf der adulten Honigbiene sowie auf deren Entwicklungstadien, indem sie die Hämolymphe absaugt. Adulte Bienen werden durch den Verlust an Hämolymphe und Sekundärinfektionen geschwächt. Die eigentlichen Schäden treten jedoch an der Bienenbrut auf. Mit zunehmendem Parasitenbefall schlüpfen vermehrt mißgebildete Bienen. Diese klinischen Erscheinungen treten meist erst nach 3 bis 4 Jahren auf. Es folgt dann oft ein rascher Zusammenbruch der Bienenvölker. Eine frühzeitige Diagnose und eine ausreichend wirksame Therapie ist daher für den Erhalt der Völker und für die Verminderung der Ausbreitungsgefahr von entscheidender Bedeutung (RITTER, 1981; DE JONG et al., 1982). 
Die Varroatose ist in der BR-Deutschland und in vielen anderen Ländern anzeigepflichtig. Den Veterinärbehörden und den Bienenhaltern steht zur Zeit weder zur Diagnose noch zur Therapie ein zugelassenes Medikament zur Verfügung.

Ein Medikament zur Bekämpfung parasitärer Milben der Honigbiene muß sehr selektiv auf Milben wirken und sollte weder die adulte Biene noch ihre Entwicklungsstadien (Ei, Made, Puppe) schädigen. Weiterhin sollte aber auch das Verhalten der Bienen, wie z. B. Bau- und Putztrieb, nicht verändert werden. Das Präparat sollte die Milben weitgehend abtöten.

In den letzten Jahren wurden zahlreiche Substanzen auf ihre Wirkung gegen Varroa-Milben überprüft (RITTER und RUTTNER, 1980 a). Neben anderen Präparaten zeigten Isopropyl-4,4'-dibrombenzilat-Suspensionen einer WP- bzw. ECFormulierung eine hohe varroazide Wirkung. Die Emulgatoren und Trägerstoffe führten jedoch besonders in starken Völkern zu erhöhten Bienenverlusten (RITTER und RutTNer, 1980 a; RenNinghoff und RitTer, 1980). Weiterhin ist die Behandlung mit Spritzmitteln sehr zeitaufwendig, da die mit Bienen besetzten Waben herausgenommen und einzeln besprüht werden müssen. Sie kann daher nur am Tag durchgeführt werden, so daß die Flugbienen von dem Mittel nicht erreicht werden. Räuchermittel dagegen zeichnen sich durch die leichte Anwendung und gute Verteilung aus. Das Räuchermittel FOLBEX (Ciba-Geigy AG) mit dem Wirkstoff Chlorbenzilat, das längere Zeit zur Bekämpfung der Akariose eingesetzt wurde, zeigte in der BR-Deutschland jedoch nur eine geringe Wirkung gegen die Varroamilben. Es lag daher nahe, einen dem FOLBEX ähnlichen Räucherstreifen mit dem stärker varroazid wirkenden Isopropyl-4, 4'-dibrombenzilat zu entwickeln. Im Labor und in Freilandversuchen mußte die Verträglichkeit und Wirkung des neuen Medikaments FOLBEX-VA überprüft werden. FOLBEX-VA wurde in früheren Publikationen FOLBEX forte genannt. Es wurde in der Bundesrepublik Deutschland unter dem Namen FOLBEX-VA-Neu zugelassen.

\section{MATERIAL UND METHODEN}

\section{Versuchspräparat und Anwendung}

Das Pränarat FOLBEX-VA wurde von der Ciba-Geigy AG in Form eines Räucherstreifens zur Verfügung gestellt. Er enthält Isopropyl-4,4'-dibrombenzilat, das chemisch zur Gruppe der Benzilsäureester gehört. Die Wirksubstanz wird beim Abglimmen des Streifens in der Beute verräuchert und wirkt so als Kontaktgift auf die Milben.

Die Anwendung erfolgte jeweils am Abend, wenn sich alle Bienen im Stock befanden. Bei Magazinbeuten wurde im aufgesetzten oberen Leerraum pro Stock ein Räucherstreifen abgeglimmt, und das Flugloch für eine Stunde geschlossen. Dies wurde 2 bis 4 mal im Abstand von 4 bzw. 7 Tagen wiederholt.

\section{Prüfzeit}

Die Prüfung erfolgte in der geplanten Anwendungszeit (Frühjahr/Herbst) und im Sommer wăhrend 
der Trachtzeit. Eine Behandlung während der Winterruhe (November bis Januar) wurde nicht durchgeführt, da bei der Anwendung von Räuchermitteln während dieser Zeit Schăden an den Bienenvölkern auftreten (z.B. Zerstörung der Wintertraube, erhöhter Nosemabefall und Einknäueln der Königin).

\section{Bestimmung der Bienenverträglichkeit}

Die Bienenvertrăglichkeit wurde anhand des allgemeinen Verhaltens der Bienen und der Bienensterblichkeit bestimmt. Die Zahl der toten Bienen konnte entweder mit Hilfe von Sperrgittern oder vor dem Stock ausgelegten Planen bestimmt werden. Die nähere Umgebung des Bienenstandes wurde fortlaufend auf tote oder verendende Bienen untersucht. Um auch eine nachhaltige Schädigung der Bienenvölker zu prüfen, wurde in Abständen ihre Entwicklung kontrolliert. Hierzu wurde die Volksstãrke anhand der von den Bienen besetzten Waben bestimmt.

\section{Bestimmung der Brutverträglichkeit}

Zur Bestimmung der Brutverträglichkeit wurden jeweils 100 Zellen, die Brut eines Alters enthielten, auf der Wabe abgesteckt. Im Abstand von 4 Tagen wurde ihre Entwicklung bis zum Schlüpfen der Bienen beobachtet. Aus der Zahl der Zellen, in denen sich die Brut nicht weiterentwickelte bzw. aus denen sie von den Bienen entfernt worden war, ergab sich die Mortalitätsrate.

\section{Bestimmung der Wirkung auf Varroamilben}

Die Wirkung auf Varroamilben wurde anhand der nach der Behandlung von den Bienen abgefallenen Milben bestimmt. Vor der Behandlung wurde hierzu in die Beute eine Bodeneinlage aus Papier mit darüber befestigtem Gitterrahmen (Maschenweite 2,4 mm) eingeschoben. Die Zahl der abgefallenen Milben wurde 24 Stunden und 4 Tage nach der Behandlung bestimmt. Zu der jeweils in den Versuchen angegebenen Zeit wurden die Bienen abgetötet und die Milben in einem Benzinbad ausgewaschen (RITTER, 1980). In Völkern, die während oder nach der Behandlung gedeckelte Brut enthielten, wurden die Brutzellen einzeln auf Varroamilben untersucht. Dabei gefundene Milben wurden dem Restmilbenbefall, d.h. der Zahl der nicht durch die Behandlung abgetöteten Milben, zugerechnet. Aus den auf den Bienen verbliebenen und den abgefallenen Milben konnte der Behandlungserfolg als Milbenmortalität in Prozent ermittelt werden.

\section{ERGEBNISSE}

\section{Prüfung der Bienenverträglichkeit}

Im Juli 1980 wurde in der Nähe von Freiburg i. Br. in 5 Völkern $4 \mathrm{mal}$ im Abstand von 7 Tagen je ein FOLBEX-VA-Räucherstreifen abgeglimmt. Unmittelbar nach den einzelnen Anwendungen verhielten sich die Bienen ruhig. Am Tag nach der Behandlung war der Bienenflug normal. Es konnten weder desorientierte Bienen noch ein gegenseitiges Ausräubern der Stöcke beobachtet werden. Im Durchschnitt starben nach den 4 Anwendungen des Räucherstreifens 51 Bienen (s = 17,9) ab. Der Unterschied zur Kontrolle mit im Durchschnitt 57 Bienen $(s=7,8)$ ist nicht signifikant (Mediantest). In 10 Monaten nach der Behandlung konnte keine nachteilige Auswirkung festgestellt werden (s. Tab. 1). In den umfangreichen Feldversuchen kam es bei der analogen Behandlung von 1200 Völkern im Frühjahr 
W. RITTER und F. PERSCHIL

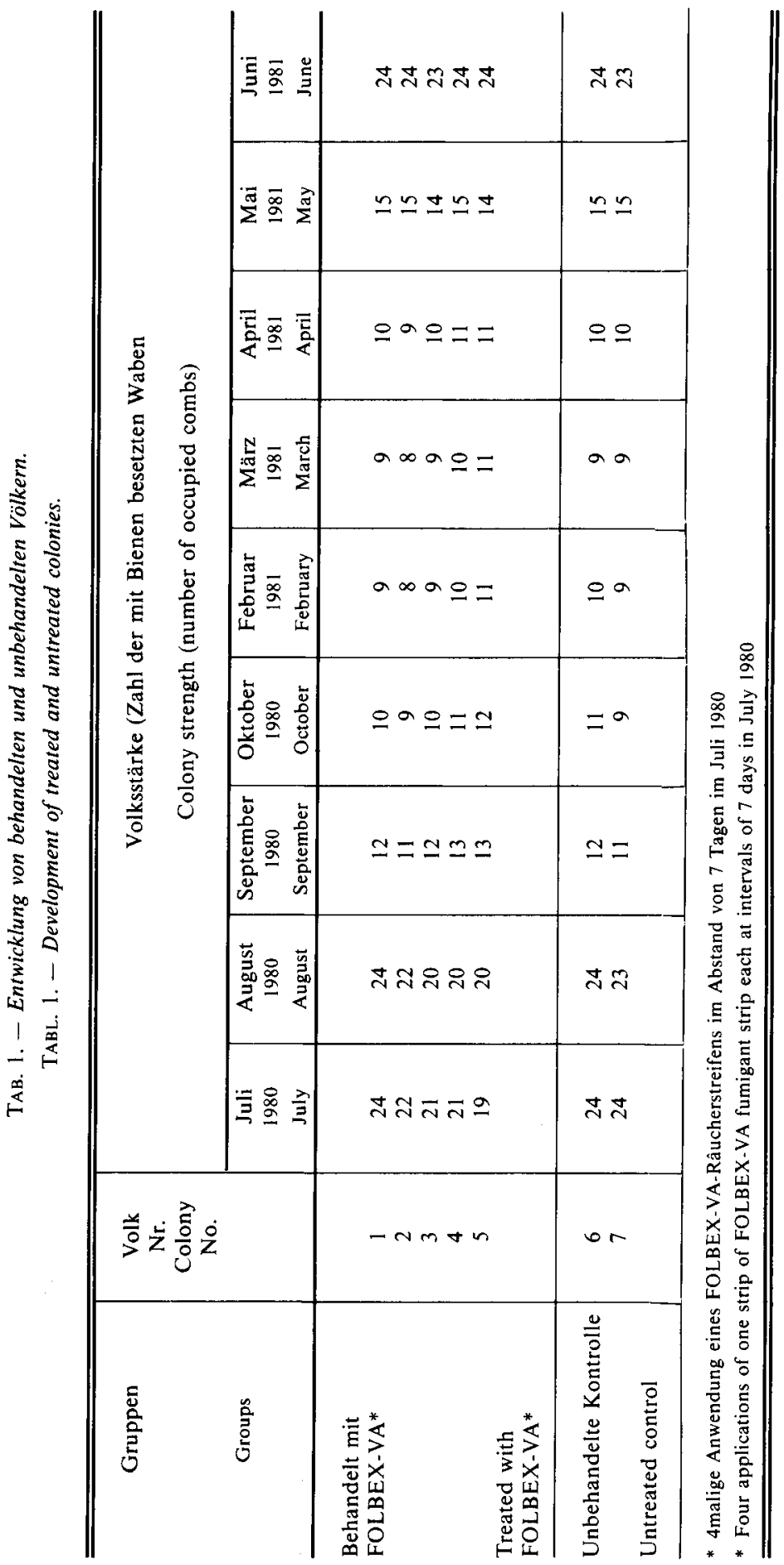


und von 1400 Völkern im Herbst zu keinen nennenswerten Bienenverlusten. Ebenso wurden nur in 3 Fällen Verluste der Königin gemeldet.

\section{Prüfung der Brutverträglichkeit}

Die Prüfung der Brutverträglichkeit erfolgte im Juli 1981 in 2 Völkern des THI-Freiburg. Eine Untersuchung zu einer anderen Jahreszeit erscheint nicht notwendig, da eine von der Saison abhängige Brutempfindlichkeit nicht bekannt ist. Die Wirkung der Behandlung mit FOLBEX-VA auf die Brut wurde an jeweils 1 bis 2 Tage alten Eiern und Larven sowie an 1 bis 4 Tage alten Puppen geprüft.

Im Vergleich zur unbehandelten Kontrolle hatte eine 4malige Anwendung von je einem FOLBEX-VA-Räucherstreifen im Abstand von 4 Tagen keinen negativen Eínfluß auf die Entwicklung der Brut in den verschiedenen Stadien (Tab. 2).

TAB. 2. - Prüfung der Brutverträglichkeit von FOLBEX-VA.

TABL. 2. - Determination of brood tolerance of FOLBEX-VA.

\begin{tabular}{|c|c|c|c|c|c|}
\hline \multirow{3}{*}{$\begin{array}{l}\text { Brutstadien } \\
\text { Brood stages }\end{array}$} & \multirow{3}{*}{$\begin{array}{c}\text { Anzahl } \\
\text { der Versuchsserien } \\
\text { mit jeweils } \\
100 \text { Brutzellen } \\
\text { Number } \\
\text { of trial series } \\
\text { with } 100 \text { brood cells } \\
\text { each time }\end{array}$} & \multicolumn{4}{|c|}{$\begin{array}{c}\text { Mortalität }(\%) \\
\text { Mortality (\%) }\end{array}$} \\
\hline & & \multicolumn{2}{|c|}{$\begin{array}{c}\text { Behandeltes Volk* } \\
\text { Treated colony* }\end{array}$} & \multicolumn{2}{|c|}{$\begin{array}{c}\text { Unbehandeltes Volk } \\
\text { (Kontrolle) } \\
\text { Untreated colony } \\
\text { (control) }\end{array}$} \\
\hline & & $\overline{\mathbf{x}}$ & $s$ & $\overline{\mathbf{x}}$ & $\boldsymbol{s}$ \\
\hline $\begin{array}{l}\text { Eier } \\
\text { Eggs }\end{array}$ & 5 & $3.4^{* *}$ & 4,4 & 2,8 & 2,9 \\
\hline $\begin{array}{l}\text { Larven } \\
\text { Larvae }\end{array}$ & 5 & $0,8^{* *}$ & 1,3 & 0,8 & 0,8 \\
\hline $\begin{array}{l}\text { Puppen } \\
\text { Pupae }\end{array}$ & 5 & $0,0^{* *}$ & - & 0,2 & 0,4 \\
\hline \multicolumn{6}{|c|}{$\begin{array}{l}\text { * 4malige Anwendung eines FOLBEX-VA-Räucherstreifens im Abstand von } 4 \text { Tagen } \\
\bar{x}=\text { Mittelwert } \\
s=\text { Standardabweichung } \\
\text { * four applications of one FOLBEX-VA fumigant strip at intervals of } 4 \text { days } \\
\bar{x}=\text { mean value } \\
s=\text { S.D. = standard deviation } \\
\text { ** Kein signifikanter Unterschied zur Kontrolle mit Hilfe des Mediantests } \\
\text { ** Difference not significant to control group by means of the median test }\end{array}$} \\
\hline
\end{tabular}




\section{Prüfung der Wirkung auf Varroamilben}

\subsection{Prüfung der Wirkung an Völkern mit Brut im Frühjahr}

Im Frühjahr 1981 wurde in Baden-Württemberg ein klinischer Feldversuch zur Prüfung von FOLBEX-VA auf Varroamilben durchgeführt. Die Völker wurden auf verschiedenen Wabenmaßen und in unterschiedlichen Beutentypen gehalten. Alle Völker enthielten während und nach der Behandlung mehrere gedeckelte Brutwaben. Von März bis Mai wurde in 1133 Völkern auf 79 Ständen je ein FOLBEX-VA-Räucherstreifen im Abstand von 7 Tagen abgeglimmt. Bei 365 Völkern der behandelten Völker konnte Varroatose festgestellt werden. In $92 \%$ dieser Völker wurden bereits nach der 1. oder 2. Anwendung des Räucherstreifens abgetötete Milben auf der Bodeneinlage gefunden. Um die nach der Behandlung auf den Bienen und in der Brut verbliebenen Milben zu bestimmen, wurde ein Teil der Völker nach 29 bzw. 72 Tagen abgetötet. Völker, die insgesamt mit mehr als 10 Milben befallen waren, hatten bereits nach der 1. oder 2. Anwendung des Räucherstreifens einen positiven Befund (s. Tabelle 3).

In 9 der 15 schwächer befallenen Völkern (5-10 Milben pro Volk) konnte die Varroatose nach der 3. oder 4. Anwendung festgestellt werden. In den übrigen Völkern dieser Gruppe, in denen bis zu 4 Milben auf Bienen und Brut verteilt waren, konnte die Varroatose auch nach 4 maliger Anwendung des Räucherstreifens nicht diagnostiziert werden.

TAB. 3. - Erste Diagnose der Varroatose mit FOLBEX-VA in abgetöteten Völkern mit Brut im Frühjahr. TABL. 3. - First diagnosis of varroa disease with FOLBEX-VA in destroyed colonies with brood in spring.

\begin{tabular}{|c|c|c|c|c|c|}
\hline \multirow[t]{2}{*}{$\begin{array}{c}\text { Gesamtzahl der } \\
\text { Milben } \\
\text { pro Volk } \\
\text { Total number } \\
\text { of mites } \\
\text { per colonies }\end{array}$} & \multirow[t]{2}{*}{$\begin{array}{l}\text { Zahl } \\
\text { der Völker } \\
\text { Number } \\
\text { of colonies }\end{array}$} & \multicolumn{4}{|c|}{$\begin{array}{c}\text { Erster positiver Befund nach der Anwendung } \\
\text { von FOLBEX-VA in Prozent der Völker } \\
\text { First positive finding after application } \\
\text { of FOLBEX-VA as percentage of colonies }\end{array}$} \\
\hline & & $\begin{array}{l}\text { 1. Anw. } \\
\text { 1st appl. }\end{array}$ & $\begin{array}{l}\text { 2. Anw. } \\
\text { 2nd appl. }\end{array}$ & $\begin{array}{l}\text { 3. Anw. } \\
\text { 3rd appl. }\end{array}$ & $\begin{array}{l}\text { 4. Anw. } \\
\text { 4th appl. }\end{array}$ \\
\hline$n$ & $n$ & $\%$ & $\%$ & $\%$ & $\%$ \\
\hline $\begin{array}{c}0 \\
<10 \\
10-100 \\
>101\end{array}$ & $\begin{array}{r}6 \\
15 \\
20 \\
9\end{array}$ & $\begin{array}{r}- \\
7 \\
90 \\
100\end{array}$ & $\begin{array}{c}- \\
27 \\
100 \\
100\end{array}$ & $\begin{array}{r}- \\
40 \\
100 \\
100\end{array}$ & $\begin{array}{c}- \\
60 \\
100 \\
100\end{array}$ \\
\hline $\begin{array}{l}\text { Insgesamt } \\
\text { Total }\end{array}$ & 50 & 64 & 75 & 80 & 86 \\
\hline
\end{tabular}

* Anwendungen von FOLBEX-VA im Abstand von 4 Tagen.

* Application of FOLBEX-VA at intervals of 4 days. 
Die Zahl der nach den einzelnen Anwendungen abgetöteten Milben war weitgehend konstant. Die Mortalitätsrate ist hier abhängig von der Zahl der während der Behandlungsperiode aus der Brut schlüpfenden Jungmilben. Der mit einer 4maligen Anwendung von je einem FOLBEX-VA-Räucherstreifen erzielte Behandlungserfolg war sehr unterschiedlich. Er schwankte zwischen 0 und $100 \%$ und betrug im Durchschnitt $59 \%$. 8 Völker, die mit bis zu 7 Milben befallen waren, wurden durch die Behandlung geheilt. Eine Abhängigkeit des Behandlungserfolges von der Befallsstärke oder von der Zahl der gedeckelten Brutzellen konnte nicht festgestellt werden (s. Tab. 4).

\section{3,2. Prüfung der Wirkung an Völkern mit Brut im Herbst}

Im September-Oktober 1980 und 1981 wurden mit Varroamilben befallene Völker mit FOLBEX-VA behandelt. Je ein Răucherstreifen wurde 4mal im Abstand von 4 Tagen in den Völkern, die mehrere gedeckelte Brutwaben enthielten, abgeglimmt. 18 der insgesamt behandelten Völker waren 2 Wochen zuvor mit Ameisensäure behandelt worden, indem mit $98 \%$ iger Ameisensäure gefüllte Dochtflaschen in die Beuten gestellt worden waren (RITTER und RUTTNER, 1980 b).

In 9 Völkern wurde der Restmilbenbefall anhand der nach einer 2maligen Anwendung von K-79 abgefallenen Milben bestimmt. Hiermit wurden, wie spätere Untersuchungen zeigten, etwa $99 \%$ der insgesamt vorhandenen Milben erfaßt.

TAB. 4. - Durchschnitlicher Behandlungserfolg bei 4maliger Anwendung eines FOLBEX-VA-Räucherstreifens im Frühjahr (mit Brut).

TABL. 4. - Mean results of treatment using 4 applications of one FOLBEX-VA fumigant strip in spring (with brood).

\begin{tabular}{|c|c|c|c|c|}
\hline $\begin{array}{c}\text { Befallsgrad } \\
\text { (Zahl der Milben } \\
\text { pro Volk) }\end{array}$ & $\begin{array}{l}\text { Anzahl der } \\
\text { Völker }\end{array}$ & $\begin{array}{l}\text { Volksstärke } \\
\text { in } 1000 \\
\text { (Durchschn.) }\end{array}$ & $\begin{array}{c}\text { Ged. Brutzellen } \\
\text { in } 1000 \\
\text { (Durchschn.) }\end{array}$ & $\begin{array}{l}\text { Behandlungserfolg } \\
\text { (Durchschn.) }\end{array}$ \\
\hline \multirow[t]{2}{*}{$\begin{array}{l}\text { Degree of infestation } \\
\text { (number of mites } \\
\text { per colony) }\end{array}$} & $\begin{array}{l}\text { Number of } \\
\text { colonies }\end{array}$ & $\begin{array}{l}\text { Strength of colony } \\
\text { in } 1000 \\
\text { (mean) }\end{array}$ & $\begin{array}{l}\text { Capped brood } \\
\text { cells in } 1000 \\
\text { (mean) }\end{array}$ & $\begin{array}{l}\text { Success of } \\
\text { treatment } \\
\text { (mean) }\end{array}$ \\
\hline & $n$ & $n$ & $n$ & $\%$ \\
\hline & 15 & $\begin{array}{l}30.9 \\
(6-42)\end{array}$ & $\begin{array}{c}10.6 \\
(3-22)\end{array}$ & $\begin{array}{c}46.0 \mathrm{~s}=45.2 \\
(0-100)\end{array}$ \\
\hline $10-100$ & 20 & $\begin{array}{l}31.1 \\
(6-46)\end{array}$ & $\begin{array}{c}10.9 \\
(1-25)\end{array}$ & $\begin{array}{c}70.9 s=21.6 \\
(18-94)\end{array}$ \\
\hline 101.1000 & 7 & $\begin{array}{l}25.9 \\
(9-44)\end{array}$ & $\begin{array}{l}13.7 \\
(8-15)\end{array}$ & $\begin{array}{c}55.3 \mathrm{~s}=20.1 \\
(29-87)\end{array}$ \\
\hline $1001-3000$ & 3 & $\begin{array}{c}28.4 \\
(18-39)\end{array}$ & $\begin{array}{c}10.9 \\
(10-12)\end{array}$ & $\begin{array}{c}53.6 s=18.3 \\
(33-69)\end{array}$ \\
\hline $\begin{array}{l}\text { insgesamt } \\
\text { Total }\end{array}$ & 45 & $\begin{array}{c}30.0 \\
(6.46)\end{array}$ & $\begin{array}{l}11.2 \\
(1.25)\end{array}$ & $\begin{array}{l}59.3 \mathrm{~s}=32.3 \\
(0-100)\end{array}$ \\
\hline
\end{tabular}

Minimum und Maximum jeweils in Klammern

Minimum and maximum given in brackets 
In allen untersuchten Völkern konnte, selbst wenn sie nur mit einer Milbe befallen waren, die Varroatose nach der 1. oder 2. Anwendung von FOLBEX-VA nachgewiesen werden. Mit der 4maligen Anwendung konnten im Durchschnitt $75 \%$ der insgesamt vorhandenen Milben abgetötet werden (s. Tab. 5). 4 schwach befallene Völker wurden durch die Behandlung geheilt, d.h. es konnten bei der anschließenden Untersuchung weder auf den Bienen noch in der Brut Milben gefunden werden. Eine Abhängigkeit des Behandlungserfolges von der Brutmenge konnte nicht festgestellt werden. Die geringere Brutzellenzahl in schwächer befallenen Völkern ist zufällig und nicht korrelliert mit dem Befallsgrad.

\subsection{Prüfung der Wirkung an Völkern ohne Brut im Herbst}

Im September-Oktober 1981 wurden im Rahmen von Feldversuchen in Baden-Württemberg und Rheinland-Pfalz bei 251 Völkern auf 16 Ständen $4 \mathrm{mal}$ im Abstand von 4 Tagen je ein FOLBEX-VA-Räucherstreifen abgeglimmt. Die Völker waren vor der Behandlung innerhalb kurzer Zeit so stark aufgefüttert worden, daß bei der kühlen Witterung im Herbst und gleichzeitig reduzierter Kastenisolation (Kaltsetzen der Völker) zum Zeitpunkt der Behandlung keine oder nur wenig Brut vorhanden war. 2 bis 3 Wochen nach der letzten Anwendung wurden 20 Völker abgetötet und auf Restmilbenbefall untersucht. In allen abgetöteten

TAB. 5. - Durchschnittlicher Behandlungserfolg bei 4maliger Anwendung eines FOLBEX-VA Räucherstreifens im Herbst (mit Brut).

TABL. 5. - Mean results of treatment using 4 applications of one FOLBEX-VA fumigant strip in autumn (with brood)

\begin{tabular}{|c|c|c|c|c|}
\hline $\begin{array}{c}\text { Befallsgrad } \\
\text { (Zahl der } \\
\text { Milben pro Volk) } \\
\text { Degree of infestation } \\
\text { (number of mites } \\
\text { per colony) }\end{array}$ & $\begin{array}{l}\text { Anzahl der } \\
\text { Völker } \\
\text { Number of } \\
\text { colonies }\end{array}$ & $\begin{array}{c}\text { Volksstärke } \\
\text { in } 1000 \\
\text { (Durchschn.) } \\
\text { Strength of colony } \\
\text { in } 1000 \\
\text { (mean) }\end{array}$ & $\begin{array}{l}\text { Ged. Brutzellen } \\
\text { in } 1000 \\
\text { (Durchschn.) } \\
\text { Capped brood } \\
\text { cells in } 1000 \\
\text { (mean) }\end{array}$ & $\begin{array}{c}\text { Behandlungserfolg } \\
\text { (Durchschn.) } \\
\text { Success of treatment } \\
\text { (mites destroyed in \%) } \\
\text { (mean) }\end{array}$ \\
\hline & $n$ & $n$ & $n$ & $\%$ \\
\hline $\begin{array}{r}<10 \\
10-100 \\
101-1000 \\
1001-5000^{*}\end{array}$ & $\begin{array}{l}6 \\
3 \\
6 \\
8\end{array}$ & $\begin{array}{c}14.8 \\
(10-16) \\
7.5 \\
(6-9) \\
9.3 \\
(6-13) \\
9.9 \\
(8-13)\end{array}$ & 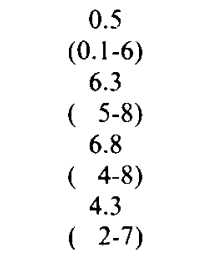 & $\begin{array}{c}83.3(s=25.8) \\
(50-100) \\
82.0(s=2.5) \\
(79-84) \\
58.7(s=26.1) \\
(17-90) \\
77.9(s=22.7) \\
(33-99)\end{array}$ \\
\hline $\begin{array}{l}\text { Insgesamt } \\
\text { Total }\end{array}$ & 23 & $\begin{array}{c}10.7 \\
(6-16)\end{array}$ & $\begin{array}{c}4.2 \\
(0.1-8)\end{array}$ & $\begin{array}{c}74.8(s=23.9) \\
(17-100)\end{array}$ \\
\hline
\end{tabular}

* 1 Volk mit 11000 Milben

* 1 colony with 11000 mites

Minimum und Maximum jeweils in Klammern

Minimum and maximum given in brackets 
TAB. 6. - Erste Diagnose der Varroatose in abgetöteten Völkern ohne Brut im Herbst.

TABL. 6. - First diagnosis of varroa disease in destroyed colonies without brood in autumn.

\begin{tabular}{|c|c|c|c|c|c|}
\hline \multirow[t]{2}{*}{$\begin{array}{c}\text { Gesamtzahl der } \\
\text { Milben } \\
\text { pro Volk } \\
\text { Total number } \\
\text { of mites per colony }\end{array}$} & \multirow[t]{2}{*}{$\begin{array}{l}\text { Zahl der Völker } \\
\text { insgesamt } \\
\text { Total number } \\
\text { of colonies }\end{array}$} & \multicolumn{4}{|c|}{$\begin{array}{c}\text { Erster positiver Befund nach der Anwendung } \\
\text { von FOLBEX-VA in Prozent der Völker } \\
\text { First positive finding after application } \\
\text { of FOLBEX-VA as percentage of colonies }\end{array}$} \\
\hline & & $\begin{array}{l}\text { 1. Anw. } \\
\text { lst appl. }\end{array}$ & $\begin{array}{l}\text { 2. Anw. } \\
\text { 2nd appl. }\end{array}$ & $\begin{array}{l}\text { 3. Anw. } \\
\text { 3rd appl. }\end{array}$ & $\begin{array}{l}\text { 4. Anw. } \\
\text { 4th appl. }\end{array}$ \\
\hline$n$ & $n$ & $\%$ & $\%$ & $\%$ & $\%$ \\
\hline $\begin{array}{c}0 \\
<10 \\
10-100 \\
>101\end{array}$ & $\begin{array}{r}4 \\
4 \\
2 \\
10\end{array}$ & $\begin{array}{r}- \\
50 \\
100 \\
100\end{array}$ & $\begin{array}{r}- \\
75 \\
100 \\
100\end{array}$ & $\begin{array}{r}- \\
75 \\
100 \\
100\end{array}$ & $\begin{array}{r}- \\
75 \\
100 \\
100\end{array}$ \\
\hline $\begin{array}{l}\text { Insgesamt } \\
\text { Total }\end{array}$ & 20 & 88 & 94 & 94 & 94 \\
\hline
\end{tabular}

Völkern, die aufgrund der Behandlung mit FOLBEX-VA einen positiven Befund hatten, waren bereits nach der 1. oder 2. Anwendung des Räucherstreifens Milben abgefallen. In einem Volk ohne Befund wurden bei der anschließenden Untersuchung 3 Milben gefunden (s. Tab. 6). Dagegen blieb in 4 weiteren Völkern der Befund negativ. Die zunächst stündliche und anschließend in größeren Zeitabständen durchgeführte Kontrolle der Bodeneinlagen ergab, daß in den ersten 11 Stunden nach der Anwendung des Räucherstreifens die meisten Milben abfallen (s. Abb.) MILBENABFALL $(\%)$

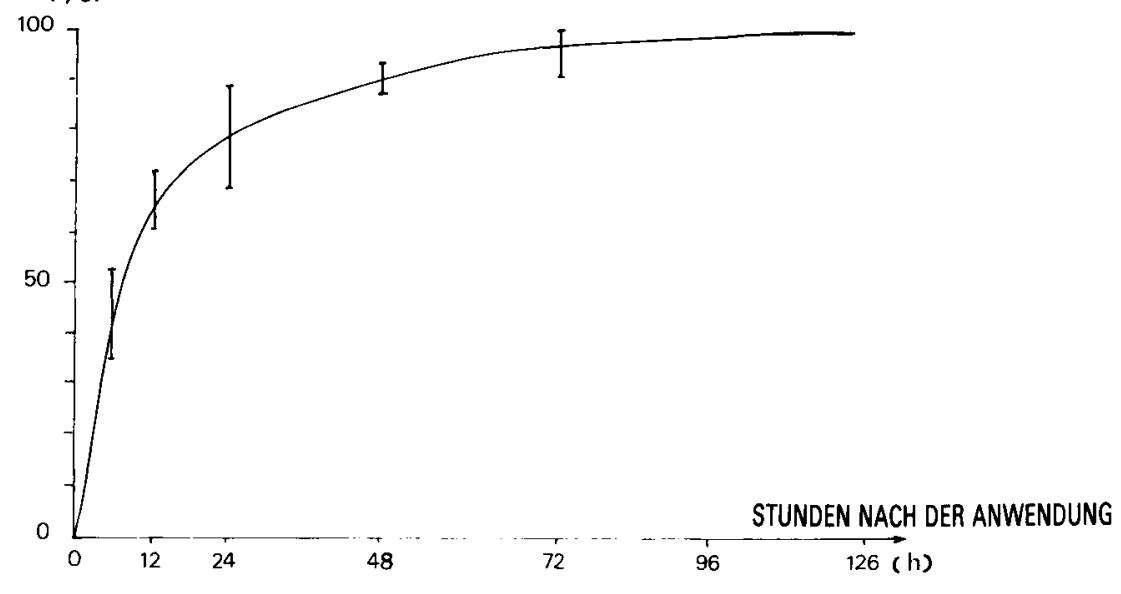

Aвв. - Milbenmortalität in Prozent vom Gesamtbefall

nach Anwendung eines FOLBEX-VA-Räucherstreifens in 10 Völkern ohne Brut.

FIG. - Mite mortality in percentage of total killed mites in 10 colonies when treated with FOLBEX-VA-smoking stripe. $x$-axis : hours after application. $y$-axis : mites falling off. 
Anhand der im Vergleich zur Kontrolle abfallenden Milben konnte eine Gesamtwirkungsdauer des FOLBEX-VA von 3 Tagen bestimmt werden.

In 2 Völkern konnte mit der 4maligen Anwendung des Räucherstreifens über $99 \%$ und in 3 weiteren alle Milben abgetötet werden. Insgesamt war der Behandlungserfolg bei 13 Völkern größer als $90 \%$. Durchschnittlich konnten $97 \%$ der in den Völkern vorhandenen Milben eliminiert werden (s. Tab. 7).

Der Behandlungserfolg konnte durch weitere 4 Anwendungen bei 6 später abgetöteten Völkern um weniger als $2 \%$ erhöht werden (s. Tab. 8).

\section{Diskussion}

\subsection{Verträglichkeit}

Bei den insgesamt 2600 Völkern, die im Frühjahr, Sommer oder Herbst mit FOLBEX-VA behandelt worden waren, kam es zu keiner erhöhten Bienensterblichkeit. Ebenso konnte unmittelbar nach und am Tag nach der Anwendung des Räucherstreifens kein verändertes Verhalten der Bienen beobachtet werden. So kann eine länger andauernde Überlagerung des Stockgeruchs ausgeschlossen werden. Die 4 malige Anwendung des FOLBEX-VA-Räucherstreifens hatte keinen Einfluß auf die Entwicklung der Brut verschiedener Altersstufen (Eier, Maden, Puppen). Die gute Brut- und Bienenverträglichkeit zeigte sich auch in den Freiland- und Feldver-

TAB. 7. - Durchschnittlicher Behandlungserfolg bei 4 maliger Anwendung eines FOLBEX-VA -Räucherstreifens im Herbst (ohne Brut).

TABL. 7. - Mean results of treatment using 4 applications of one FOLBEX-VA-fumigant strip in autumn (without brood).

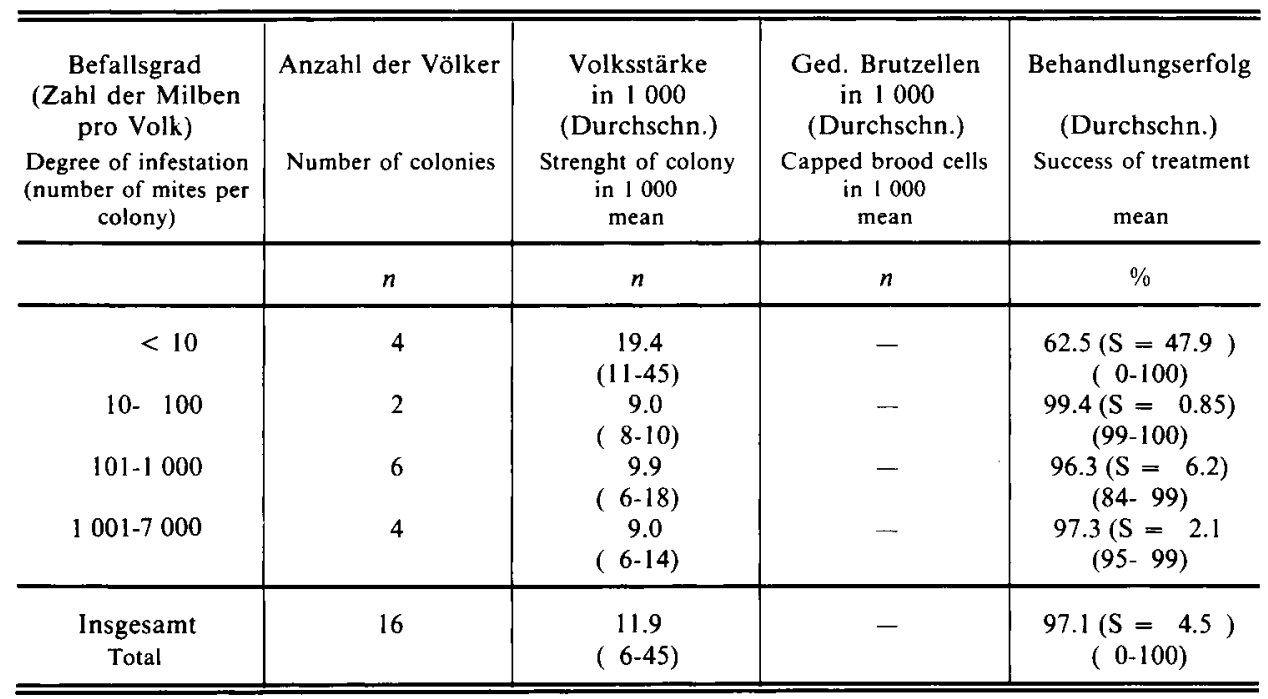

Minimum und Maximum jeweils in Klammern.

Minimum and maximum given in brackets. 
FOLBEX-VA GEGEN VARROAMILBEN

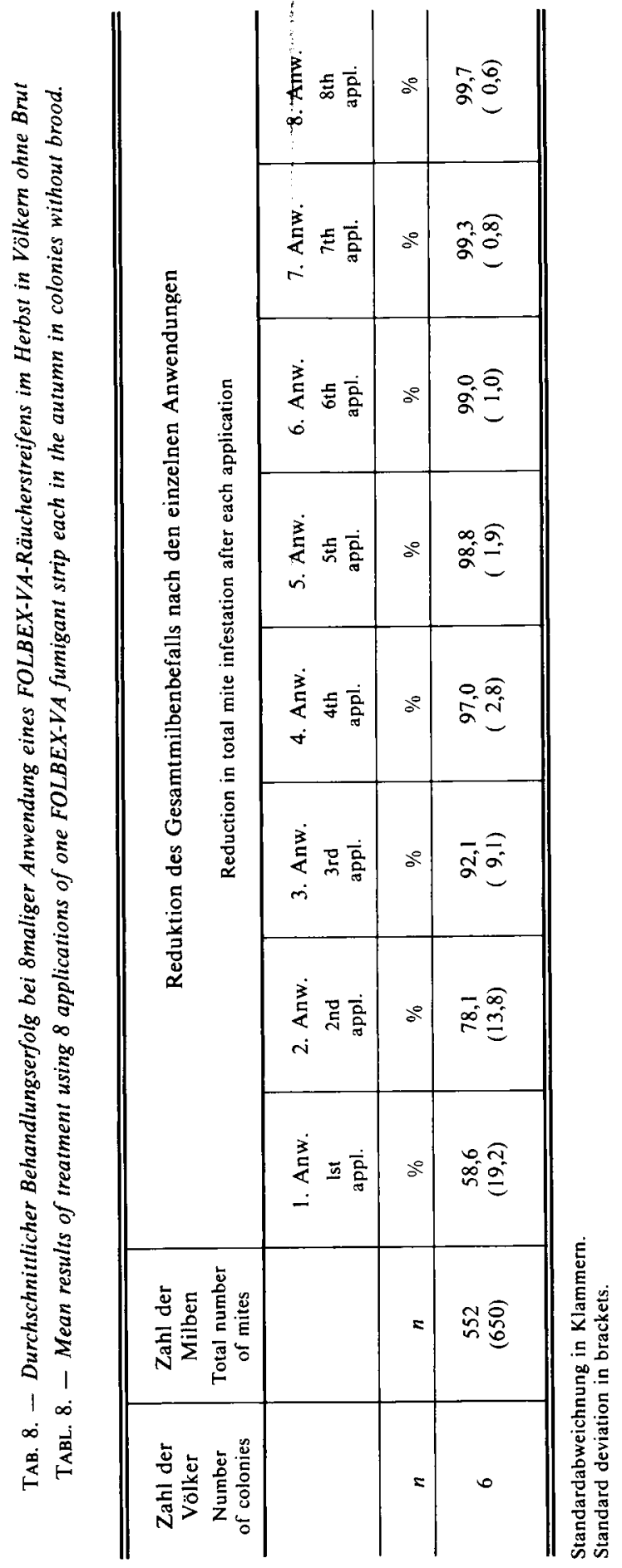


suchen an der ungestörten Entwicklung der Bienenvölker. Während der Beobachtungszeit von bis zu 10 Monaten nach der Behandlung konnte bei 5 Völkern im Vergleich zur unbehandelten Kontrollgruppe (2 Völker) keine unterschiedliche Entwicklung der Bienenvölker beobachtet werden. Ebenso konnten alle bisher behandelten Völker erfolgreich ausgewintert werden. Im Gegensatz zu den früher bei der Behandlung mit FOLBEX (Wirkstoff : Chlorbenzilat) beobachteten Königinnenverlusten traten diese mit FOLBEX-VA nicht auf. In allen Versuchen erwies sich FOLBEX-VA somit als gut bienen- und brutverträglich.

\subsection{Diagnose}

In einem klinischen Feldversuch im Frühjahr konnte nach der Behandlung mit FOLBEX-VA in 365 Völkern Varroatose festgestellt werden. In $92 \%$ der Völker wurde die richtige Diagnose bereits nach der 1. oder 2. Anwendung des Räucherstreifens festgestellt. Hierbei fielen innerhalb von 11 Stunden nach der Anwendung des Räucherstreifens die jeweils meisten Milben ab.

Die Ergebnisse der zur Restmilbenbestimmung abgetöteten Völker zeigen, daß bei einem negativen Befund nach der 2 maligen Anwendung des Medikaments im Frühjahr ein Befall von weniger als 10 Milben vorliegen kann. Wird Varroatose auch nach 2 weiteren Anwendungen des Räucherstreifens nicht erkannt, so können bis zu 4 Milben in den Völkern verteilt sein. Da alle Völker während der Behandlung zahlreiche Brutwaben enthielten, konnten diese Milben vermutlich in den gedeckelten Brutzellen überleben. Ebenso ist eine Neuinfektion durch stark befallene Nachbarvölker möglich. Im Herbst konnte nach 2 Anwendungen von FOLBEX-VA in Völkern mit Brut die Varroatose selbst bei einem Befall mit nur einer Milbe nachgewiesen werden. Es kann hierbei jedoch nicht ausgeschlossen werden, daß die Vorbehandlung mit Ameisensäure einen Einfluß auf das Versuchsergebnis hatte. In Völkern ohne Brut war der Befund in allen mit mehr als 3 Milben befallenen Völkern nach der 2. Anwendung positiv. Nur in einem schwächer befallenen Volk konnte dies selbst nach der 4. Anwendung des Räucherstreifens nicht festgestellt werden.

Mit Hilfe einer 2maligen Anwendung je eines FOLBEX-VA-Räucherstreifens im Abstand von 4 Tagen kann somit sowohl im Frühjahr als auch im Herbst in Völkern mit Brut ein Befall von mehr als 5 Milben sicher nachgewiesen werden.

\subsection{Therapie}

Zur Prüfung des Therapieerfolges wurden nach der Behandlung 84 Völker abgetötet. In Völkern mit Brut konnten im Frühjahr bzw. Herbst nur sehr schwach befallene Völker ( $<10$ Milben) geheilt werden. In Völkern ohne Brut war dies auch bei einem nur wenig stärkeren Befall ( $<100$ Milben) möglich. Nur in schwächer befallenen Völkern besteht somit eine Chance der Heilung. Vermutlich halten sich die Milben überwiegend auf den adulten Bienen und nicht in der Brut auf. Bei allen übrigen Völkern ist der Behandlungserfolg in den schwach befallenen Gruppen 
(10-100 Milben) höher als in stärker befallenen Gruppen. In den Gruppen mit einem Befallsgrad unter 10 Milben wird wegen der geringen Milbenzahl ein insgesamt schlechtes Behandlungsergebnis vorgetäuscht. Der Erfolg der Therapie hängt somit von dem Befallsgrad der Völker ab. Er ist in Völkern mit gedeckelter Brut sehr unterschiedlich. Der im Herbst im Vergleich zum Frühjahr um im Durchschnitt $16 \%$ höhere Behandlungserfolg zeigt eine größere Empfindlichkeit der Milben im Herbst an. Dies konnte auch bei anderen Medikamenten beobachtet werden (MORITZ, 1981).

In Völkern mit Brut kann durch eine Behandlung im Frühjahr oder im Herbst der Befall deutlich gesenkt werden. Die unter den Deckeln der Brutzellen geschützten Milben können von dem Medikament - wie auch von anderen bekannten Applikationsformen und Medikamenten - nicht erreicht werden (RUTTNER und RITTER, 1981).

Da diese Milben nur subletale Dosen an Wirkstoff aufnehmen, besteht mit der Häufigkeit der Anwendung die Gefahr der Selektion von resistenten Varroamilben. Waren die Völker während der Behandlung im Herbst brutfrei, so konnten im Durchschnitt $97 \%$ der insgesamt vorhandenen Milben abgetötet werden. Eine Erhöhung der Zahl der Anwendungen auf bis zu 8 konnte den Behandlungserfolg nur unwesentlich vergrößern. In 3 der 16 untersuchten Völker konnten sämtliche Milben abgetötet werden (3. Tab. 9). Auch für FOLBEX-VA gilt somit, daß der Behandlungserfolg in Völkern mit wenig oder keiner Brut größer ist als in Völkern mit Brut. Im Frühjahr kann die Brut in Wirtschaftsvölkern weder reduziert noch aus

TАВ. 9. - Bekämpfung der Varroatose durch 4malige Anwendung von FOLBEX-VA.

TABL. 9. - Control of varroa disease with 4 applications of FOLBEX-VA.

\begin{tabular}{|c|c|c|c|c|}
\hline $\begin{array}{c}\text { Zeitpunkt der } \\
\text { Behandlung } \\
\text { (Jahreszeit) } \\
\text { Time of treatment } \\
\text { (season) }\end{array}$ & $\begin{array}{l}\text { Zustand der } \\
\text { Völker } \\
\text { State of the } \\
\text { colonies }\end{array}$ & $\begin{array}{l}\text { Anzahl der } \\
\text { Völker } \\
\text { No. of } \\
\text { colonies }\end{array}$ & $\begin{array}{c}\text { Durchschnittliche } \\
\text { Zahl der abgetöteten } \\
\text { Milben } \\
\text { in Prozent } \\
\text { Average No. of } \\
\text { killed mites } \\
\text { in percentage }\end{array}$ & $\begin{array}{c}\text { Zahl der geheilten } \\
\text { Völker } \\
\text { in Prozent } \\
\text { No. of completely } \\
\text { cleared colonies } \\
\text { in percentage }\end{array}$ \\
\hline $\begin{array}{l}\text { Frühjahr } \\
\text { Spring }\end{array}$ & $\begin{array}{l}\text { mit Brut } \\
\text { with brood }\end{array}$ & 45 & $59,3 \%(0-100)$ & $18 \%$ \\
\hline $\begin{array}{l}\text { Herbst } \\
\text { Autumn }\end{array}$ & $\begin{array}{l}\text { mit Brut } \\
\text { with brood }\end{array}$ & 23 & $74,8 \%(17-100)$ & $17 \%$ \\
\hline $\begin{array}{l}\text { Herbst } \\
\text { Autumn }\end{array}$ & $\begin{array}{l}\text { ohne Brut } \\
\text { without brood }\end{array}$ & 16 & $97,1 \%(0-100)$ & $19 \%$ \\
\hline
\end{tabular}

In Klammern wird der jeweils höchste und niedrigste Wert angegeben.

In brackets : the highest and lowest values in each case. 
ihnen entfernt werden, da wegen des Abgangs der Winterbienen eine neue Bienengeneration notwendig ist, um das Volk am Leben zu halten. Im Herbst nimmt dagegen die Bruttätigkeit zunehmend ab. Dies kann durch Maßnahmen wie starkes Auffüttern und Kaltsetzen (Reduktion der Kastenisolation) unterstützt werden. Die Entnahme der Restbrut hat dann meist keine negative Auswirkung auf die Bienenvölker. Eine erfolgreiche Therapie kann somit nur in brutfreien Völkern im Herbst durchgeführt werden.Da die Bruttätigkeit nur über einen relativ kurzen Zeitraum eingeschränkt werden kann und das Medikament eine 3 Tage andauernde akarizide Wirkung besitzt, sollte es 4-mal im Abstand von 4 Tagen angewandt werden.

Nur bei der Behandlung der Bienen im Kunstschwarm war die Mortalitätsrate der Milben noch höher als in den hier beschriebenen Versuchen (PFEFFERLE und RITTER, 1982). Bereits nach einer zweimaligen Anwendung je eines FOLBEX-VARäucherstreifens im Abstand von 24 Stunden konnten 4 Schwärme geheilt und in einem weiteren $98 \%$ der Milben abgetötet werden. Im Kunstschwarm wird der Rauch offensichtlich noch gleichmäßiger verteilt als im Volk auf Waben und die Milben haben noch weniger Möglichkeit Unterschlupf zu finden. Eine $100 \%$ ige Abtötung und damit Ausrottung der Milben ist nach bisherigen Kenntnissen jedoch bei einer Parasitose nicht möglich. So ist auch bei einem guten Mittel damit zu rechnen, daß einige Milben überleben können (WACHENDÖRFER et al, 1981).

\subsection{Schlußbemerkungen}

In den durchgeführten Versuchen konnte für FOLBEX-VA eine ausreichend hohe varroazide Wirkung bei gleichzeitig guter Bienen- und Brutverträglichkeit nachgewiesen werden. Es erfüllt somit die Ansprüche, die an ein derartiges Präparat gestellt werden.

FOLBEX-VA wurde vom Bundesgesundheitsamt (BGA) der Bundesrepublik Deutschland im Jahr 1982 unter dem Namen "FOLBEX-VA-Neu " zur Bekämpfung der Akariose und Varroatose sowie zur Diagnose der Varroatose zugelassen. Die Zulassung erfolgte nach den für Tierarzneimittel üblichen Voraussetzungen, d.h. einer klinischen Prüfung und umfangreicher toxikologischer Tests sowie Rückstandsuntersuchungen.

FOLBEX-VA darf -wie alle Medikamente- nur dann angewandt werden, wenn kein Honig gewonnen wird, der dem menschlichen Verzehr dienen soll. Die Qualität eines später gewonnen Honigs wird in keiner Weise beeinträchtigt. 


\title{
SUMMARY
}

\author{
DETERMINATION OF ACTION OF FOLBEX-VA \\ (ISOPROPYL-4, 4'-DIBROMOBENZILATE) \\ AGAINST VARROAMITES AND OF BEE TOLERANCE
}

\section{Introduction}

Varroa disease can so debilitate bee colonies that honey yields and pollination performance decline and untreated colonies could die (RITTER, 1981; DE JONG et al, 1982).

The causal agent of varroa disease, the mite, Varroa jacobsoni Oudemans, occurs on the adult bee as well as in the brood. After a latent period of 3 to 4 years, a colony may fail to develop normally. Since 1977 , the varroacidal effect and the bee-tolerance of numerous substances have been tested in the Federal Republic of Germany (RITTER und RUTTNER, $1980 \mathrm{a}$ ).

Isopropyl-4, 4-dibromobenzilate is the active ingredient of FOLBEX-VA*, which was developed by CIBA-GEIGY Ltd. in collaboration with the Veterinary Institute, Freiburg, and has been approved by the German Federal Health Office (BGA) as a veterinary drug for the diagnosis of bees for varroamite infestation and for the control of mite infestation in bees, caused by the varroa mite (varroa disease) or the acarine mite (acarine disease).

\section{Material and methods}

\subsection{Trial preparation}

Isopropyl-4, 4-dibromobenzilate is the active substance in the FOLBEX-VA fumigant strips manufactured by CIBA-GEIGY Ltd. The substance is vaporized as the strip smoulders in the hive and acts as a contact poison on the mites.

\subsection{Application}

In field tests the strips are used in the evening when all the bees are inside the hive. The treatment should not be applied during the day to avoid the risk of robbing. At the time of application, the outside temperature should not exceed $10^{\circ} \mathrm{C}$ and there should be no risk of night frost. Food must be available to the bees during the treatment. For this purpose, honeycombs are uncapped or sugar syrup is given before the treatment. Furthermore, during the fumigation the bees need additional space so they can move about. In multiple story hives an empty chamber is set aside for this purpose and in side-opening hives the window is pulled back or the crown board to the honey chamber removed. The fumigant strip is burned in the empty upper chamber.

\subsection{Determination of bee and brood tolerability}

Bee tolerance was determined on the basis of the behaviour and mortality of the bees. The dead bees were collected in containers placed in front of the hive entrance. The development of treated and untreated colonies was observed over a period of 2 years. For the determination of brood tolerance the development of groups of 100 cells each, with brood of different stages, was kept under observation until emergence of the imagoes.

2.4. Determination of the effect on varroa mites

The effect on varroa mites was determined on the basis of the number of mites falling off and the number remaining on the bees after the treatment. For this purpose the bees were killed at the end of the trial and the mites washed off in benzine.

\section{Results and discussion}

\subsection{Tolerance}

To date about 2,600 colonies have been treated with the new drug. There was no increase in bee mortality either in spring or in autumn. Nor was any change observed in the behaviour of the bees, either

* Introduced in the German Federal Republic under the name FOLBEX VA NEU. The preparation has been described in previous publications as " Folbex forte". 
immediately or the day after application of the fumigant strip. The treated colonies developped normally during the 12 month observation period and were wintered successfully. The treatment had no effect on the development of the brood. In contrast to the queen losses observed earlier using FOLBEX (active principle : chlorobenzilate), none occured following the use of FOLBEX-VA. In all the tests the new drug proved safe for adult bees and brood.

\subsection{Diagnosis and treatment of varroa disease}

In the autumns of 1980 and 1981 and also in the spring of 1981, field trials were carried out in the Federal Republic of Germany (Baden-Württemberg and Rheinland-Pfalz) to study the effect of the FOLBEX-VA on varroa mites. In order to ascertain the diagnostic accuracy and the therapeutic success of the drug, 84 colonies were killed. The bees were washed out in benzine and the capped brood cells examined individually for mite infestation. In colonies with a total infestation of more than 10 mites, the varroa disease could be detected after two applications of the fumigant strip, irrespective of the age of the brood and the season when the diagnosis was made. If the colonies were infested with fewer than 10 mites, the mites could also be detected after 2 applications. With infestations of less than 5 mites, varroa disease could only be detected after a third or fourth application, or in a few cases not at all (see Table 3 and 6).

The results show that with two applications of FOLBEX-VA fumigant strips at 4-day intervals an infestation of more than 5 mites can be reliably detected even in colonies with brood.

With four applications of the fumigant strip in colonies with sealed brood $59 \%$ of all the mites present could be killed in the spring and $75 \%$ in the autumn (see Table 4 and 5). The approximately $16 \%$ higher success rate in the autumn indicates a greater sensitivity of the mites at this time of the year. If no brood was present in the colonies during the treatment, $97 \%$ of the mites could be killed (see Table 7). It is thus true for FOLBEX-VA - and other similar substances - that a successful treatment is possible only in broodless colonies, in the autumn. The most favourable time for the treatment is in the months of September and October. The brood can be reduced in the colonies, for example by heavy feeding for a short time, when the bees are preparing for winter. These colonies should be kept less insulation, when the feeding is completed.

In some of the colonies all the mites could be eliminated. These were mostly colonies with low infestations (see Table 9). The differences between colonies with and without brood were minute. In colonies when low infestation the mites are presumably confined mainly to the adult bees. An even greater therapeutic success was obtained, irrespective of the degree of infestation, when the treatment of bees was made artificial swarms. After only 2 applications of the fumigant strip, with an interval of 24 hours, four swarms were completely cleared and in another cases $98 \%$ of the mites were killed. In the artificial swarm, the smoke apparently has a better distribution than in colonies with combs and the mites are exposed and more vulnerable.

From these results, the treatment of varroa disease is best applied in the autumn, when the colonies have less brood. The treatment requires 4 applications each of one fumigant strip, at 4-days intervals. With prosper use, there is no danger of resistant strains of mites developing in the foreseeable future. According to our present knowledge, in a parasitosis, a $100 \%$ killing of the mites, is not possible. FOLBEX-VA thus fulfills the requirements demanded of a drug for the control of parasitic bee-mites.

\section{RÉSUMÉ \\ DÉTERMINATION DE L'ACTION DE FOLBEX-VA (ISOPROPYL-4,4'-DIBROMOBENZILATE) \\ CONTRE L'ACARIEN VARROA ET DE SA TOLERANCE PAR LES ABEILLES}

\section{Introduction}

La varroase peut affaiblir les colonies d'abeille à un tel point que les récoltes de miel et les performances de pollinisation régressent et que les colonies non traitées dépérissent (RITTER, 1981; DE 
JoNG et al., 1982). Depuis 1977 on a testé en République fédérale d'Allemagne l'action varroacide et la toxicité vis-à-vis des abeilles de nombreuses substances (RITTER et RUTTNER, 1980 a).

L'isopropyl-4, 4'-dibromobenzilate est la substance active du FOLBEX-VA, qui a été mis au point et expérimenté par la firme Ciba Geigy en collaboration avec l'Institut vétérinaire de Fribourg. Il a été agréé par l'Office fédéral de la Santé (BGA) comme produit vétérinaire pour diagnostiquer une attaque de Varroa et traiter la varrosse et l'acariose.

\section{Matériel et méthodes}

\subsection{Préparation testée}

Le FOLBEX-VA* est administré sous forme de tickets fumigènes. En se consumant à l'intérieur de la ruche, ils dégagent des vapeurs de la substance active (isopropyl-4, 4'-dibromobenzilate) qui agit comme toxique de contact sur les acariens.

\subsection{Application}

Les tickets sont administrés le soir lorsque toutes les abeilles sont rentrées à la ruche. Le traitement ne doit pas être effectué durant la journée en raison des risques de pillage. La température extérieure au moment du traitement doit être supérieure à $10^{\circ} \mathrm{C}$ et le risque de gelée nocturne absent. Pendant le traitement il faut que les colonies disposent de nourriture ad libitum. Pour cela il est nécessaire de fournir auparavant des rayons de miel désoperculés ou du sirop. De plus pendant l'administration de tickets fumigènes les abeilles ont besoin d'espace supplémentaire où se déplacer. Dans les ruches à hausses multiples on place un cadre vide; dans les ruches à ouverture latérale on ôte la fenêtre ou bien on déplace le couvre-cadres dans la chambre à miel. Le ticket fumigène est brûlé dans la chambre supérieure vide. Le médicament ne doit être utilisé qu'en dehors de la période de miellée afin d'éviter des résidus dans le miel.

2.3. Détermination de la tolérance par les abeilles et le couvain

La tolérance par l'abeille a été déterminée d'après leur comportement et leur mortalité. On a récolté les abeilles mortes dans des récipients placés devant l'entrée de la ruche et observé durant 2 années le développement de colonies traitées et non traitées. Pour déterminer la tolérance par le couvain on a observé jusqu'à l'éclosion des adultes le développement de groupes de 100 cellules renfermant du couvain à des stades différents.

\subsection{Détermination de l'action sur l'acarien Varroa}

Elle repose sur le nombre d'acariens tombés et le nombre d'acariens restant sur les abeilles après le traitement. Pour cela on a tué les abeilles en fin d'expérience et éliminé les acariens dans un bain de benzine.

\section{Résultats et discussion}

\subsection{Tolérance}

On a traité environ 2600 colonies avec le médicament. Il n'y a pas eu d'accroissement de la mortalité ni au printemps, ni à l'automne. Aucun changement non plus n'a été observé dans le comportement des abeilles, que ce soit juste après l'administration du ticket fumigène ou un jour plus tard. Les colonies traitées se sont développées normalement durant les 12 mois d'observation et ont hiverné avec succès. Le traitemenent n’a eu aucun effet sur le développement du couvain à ses divers stades. Contrairement à ce qui s'était passé avec le traitement au FOLBEX (principe actif : chlorobenzilate), il n'y a eu aucune perte de reines avec l'utilisation de FOLBEX-VA. Les tests ont donc tous prouvé que le nouveau médicament était bien toléré à la fois par les abeilles et par le couvain.

\subsection{Diagnostic et traitement de la varroose}

Des expérimentations en champ ont été menées dans le Bade-Wurtemberg et la Rhénanie-Palatinat (RFA) au cours des automnes 1980 et 1981 et du printemps 1981 afin d'étudier l'action du FOLBEX-VA

* Agréé en RFA sous le nom de FOLBEX-VA-NEU et mentionné dans les précédentes publications sous le nom de « Folbex forte». 
sur Varroa. Pour s'assurer de la précision du diagnostịc et de l'efficacité thérapeutique, on a tué 84 colonies. Les abeilles ont été lavées dans de la benzine et les cellules de couvain operculées examinées individuellement. Dans les colonies dont l'infestation était supérieure à 10 acariens, la varroase a pu être détectée après administration de 2 tickets fumigènes, quels que soient le stade du couvain et de la période de l'année auxquels la maladie avait été diagnostiquée. Si le nombre de Varroa dans la colonie était inférieur à 5 , on n'a pu détecter la maladie qu'après une $3^{\mathrm{e}}$ ou une $4^{\mathrm{e}}$ application et même dans certains cas elle n'a pas pu être du tout diagnostiquée (cf. Tabl. 3 et 6 ).

Les résultats montrent que deux applications de tickets fumigènes à 4 jours d'intervalle permettent de diagnostiquer de façon sûre une infestation de plus de 5 acariens, même dans des colonies avec couvain.

Avec 4 applications dans des colonies ayant du couvain operculé, il a été possible de tuer $59 \%$ des acariens présents au printemps et $75 \%$ à l'automne (cf. Tabl. 4 et 5). L'efficacité accrue de $16 \%$ à l'automne indique une plus grande sensibilité des acariens à cette période de l'année. Lorsqu'il n'y avait pas de couvain dans la colonie au moment du traitement, $97 \%$ des acariens ont pu être tués (cf. Tabl. 7). Un traitement au FOLBEX-VA - comme avec toutes les substances semblables connues - n'est pleinement efficace que dans des colonies dépourvues de couvain, à l'automne. La période la plus favorable pour le traitement est septembre et octobre. Par des mesures telles que nourrissement intensif et abaissement de la température il est possible d'obténir au moins à court terme des colonies sans couvain.

Dans certaines colonies tous les acariens ont pu être éliminés. Il s'agissait essentiellement de colonies à faible infestation (cf. Tabl. 9). Dans ce cas les différences entre colonies avec et sans couvain ont été faibles. Dans les colonies faiblement infestées il est vraisemblable que les acariens sont confinés principalement aux abeilles adultes. Un succès thérapeutique plus grand n'a pu être obtenu, quel que soit le degré d'infestation, que dans le traitement des abeilles d'un essaim artificiel. Après 2 applications de ticket fumigène, à $24 \mathrm{~h}$ d'intervalle, 4 essaims ont été totálement débarrassés des acariens et dans un autre essaim $98 \%$ d'entre eux furent tués. Il semble que la fumée est mieux répartie dans un essaim artificiel que dans des colonies sur cadres et que les acariens ont moins de possibilités de se cacher.

D'après ces résultats il est préférable d'entreprendré le traitement de la varroose à l'automne, lorsque les colonies ont moins de couvain. Le traitement consiste en 4 applications d'un ticket fumigène à 4 jours d'intervalle. Une utilisation correcte ne doit pas donner lieu dans un proche avenir au développement de souches résistantes. Selon nos connaissances actuelles il n'est pas possible d'exterminer les acariens en cas de parasitose. FOLBEX-VA remplit donc les conditions requises d'un médicament pour lutter contre les acariens parasites de l'abeille.

\section{LITERATURVERZEICHNIS}

De Jong, Morse R. A. und Eickwart G. C., 1982. - Mite pests of honeybees. Ann. Rev. Entomol., 27, 229-252.

MORITż RF. A., 1980. - Altersabhängige Empfindlichkeit von Varroa jacobsoni Oud. gegen K-79 (Chlordimeformhydrochlorid), pp. 62-68 aus Diagnose und Therapie der Varroatose. Bukarest, Rumänien : Apimondia Verlag.

PFEFFERLE K. und RITTER W., 1982. - Versuche zur varroafreien Jungvolkbildung mit dem « Ruck-Zuck "-Kästchen. Allg. dtsch. Imkerztg., 5, 65-67.

RenNinghofF V. und RitTeR W., 1980. - Erfahrungen mit der Varroatose-Therapie in Tunesien. Allg. disch. Imkerztg., 5, 65-67.

Ritrer W., 1980. - Zur Methodik der Prüfung von Chemotherapeutika zur Bekămpfung der Varroatose der Honigbiene. Apidologie 11 (2), 131-141.

RITTER W., 1981. - Varroa disease of the honeybee Apis mellifera. Bee world, 62 (4), 141-153.

Ritter W. und RutTnER F., 1980 a. - Chemotherapie. Allg. dtsch. Imkerztg., 5, 138-146. 
RitTer W. und RUTTNER F., 1980 b. - Ameisensäure-Labor- und Freilandversuche. Allg. dtsch. Imkerztg., 5, 151-155.

Ruttner F. und RitTer W., 1981. -Eine Methode zur Varroatosebehandlung über das Futter. Apidologie, 12 (1), 75-77.

Wachendörfer G., Valder W. A., Kaiser E., Maul V., Wissen W., Ruttner F., Harlander P., Becker W., Botrin F., 1981. - Erfahrungen mit dem Akarizit K-79 (Chlordimeformhydrochlorid) in Hessen zur Bekämpfung der Varroatose der Honigbiene. Dtsch. tierärztl. Wschr., 88, 161-208. 